\title{
BMJ Open Global prevalence and incidence of surgical site infections after appendectomy: a systematic review and meta-analysis protocol
}

\author{
Celestin Danwang, ${ }^{1}$ Temgoua Ngou Mazou, ${ }^{2}$ Joel Noutakdie Tochie, ${ }^{1}$ \\ Rolf Nyah Tuku Nzalie, ${ }^{3}$ Jean Joel Bigna ${ }^{4,5}$
}

To cite: Danwang C, Mazou TN, Tochie JN, et al. Global prevalence and incidence of surgical site infections after appendectomy: a systematic review and metaanalysis protocol. BMJ Open 2018;8:e20101. doi:10.1136/ bmjopen-2017-020101

- Prepublication history and additional material for this paper are available online. To view please visit the journal (http:// dx.doi.org/10.1136/bmjopen2017-020101).

Received 13 0ctober 2017 Revised 4 June 2018 Accepted 9 August 2018
Check for updates

(C) Author(s) (or their employer(s)) 2018. Re-use permitted under CC BY-NC. No commercial re-use. See rights and permissions. Published by BMJ.

For numbered affiliations see end of article.

Correspondence to Dr Celestin Danwang; danram07@yahoo.fr

\section{ABSTRACT}

Introduction Acute appendicitis is a surgical emergency and the most frequent aetiology of acute surgical abdominal pain in developed countries. Universally, its widely approved treatment is appendectomy. Like all surgical procedures, appendectomy can be associated with many complications among which are surgical site infections (SSIs). Despite the increasing number of appendectomies done around the world and the associated morbidities related to SSI after appendectomy, there is still scarcity of data concerning the global epidemiology of SSI after appendectomy. The current review aims at providing a summary of the published data on epidemiology of SSI after appendectomy.

Methods and design We will include randomised controlled trials, cohort studies, case-control and crosssectional studies. Electronic databases including Embase, MEDLINE and ISI Web of Science (Science Citation Index) will be searched for relevant abstracts of studies published between 1 January 2000 and 30 December 2017, without language restriction. The review will be reported according to the Preferred Reporting Items for Systematic Reviews and Meta-Analyses guidelines. After screening of abstracts, study selection, data extraction and assessment of risk of bias, we shall assess the studies individually for clinical and statistical heterogeneity. Appropriate metaanalytic techniques will then be used to pool studies judged to be clinically homogenous. Visual inspection of funnel plots and Egger's test will be used to detect publication bias. Results will be presented by country and continent.

Ethics and dissemination Since primary data are not collected in this study, ethical approval is not required. This review is expected to provide relevant data to help in quantifying the global burden of SSI after appendectomy. The final report will be published in a peer-reviewed journal.

Trial registration number CRD42017075257.

\section{INTRODUCTION}

Defined as an acute inflammation of the appendix, acute appendicitis is the most frequent aetiology of acute surgical abdominal pain in developed countries. ${ }^{1}$ Its currently
Strengths and limitations of this study

- To the best of our knowledge, this will be the first global systematic review summarising contemporary data on the occurrence of surgical site infection (SSI) after appendectomy.

- This review will be conducted with strong and robust methodological processes and statistical analyses to help in providing the highest level of evidence that will help to acquire a better evidence-based decision making on this topic.

- A limited number of studies on the subject in low-income and middle-income countries could lead to an underestimation of the burden of SSI in this specific part of the world.

approved standard of treatment is appendectomy. ${ }^{23}$ In the USA, the annual number of people undergoing appendectomy in acute care hospital is estimated at $300000 .^{4-6}$

Appendectomy can be performed through a laparoscopic or an open surgery technique, with laparoscopy being the most recommended method. ${ }^{7-10}$ This is because the former is associated with reduced postoperative pain, a short length of hospital stay with a subsequent earlier return to day-to-day activities, reduced postoperative ileus and better cosmetic results. ${ }^{7-10}$ Within the last five decades, the mortality associated with acute appendicitis has drastically dropped from $26 \%$ to less than $1 \% .^{1112}$

Like all surgical procedures, appendectomy can be associated with several postoperative complications like persistent ileus, cecal fistula, pelvic or abdominal abscess and surgical site infections (SSI). ${ }^{13}$ SSIs are associated with a prolonged postoperative morbidity, which is a substantial additional healthcare cost, making this complication a concern for all surgical teams. ${ }^{14} 15$ Some studies done in Brazil, Sweden, China and 
the USA report SSI prevalence rates of $7.2 \%, 5.9 \%, 6.2 \%$ and $2.9 \%$, respectively, after appendectomy. ${ }^{16}$ Moreover, a recent systematic review on SSIs after appendectomy performed in low and middle Human Development Index countries (LMHDICs) ${ }^{17}$ found a high rate of SSI in LMHDICs compared with data of isolated studies done in high Human Development Index countries. The aforementioned systematic review differs from the review we plan to do by the fact that our systematic review will integrate data from developed and developing countries; hence, this will help provide a global estimation of the burden posed by SSI after appendectomy. In addition, our systematic review will be associated with meta-analysis.

Faced with this gap in the contemporary literature, it is evident that there is still scarcity of data regarding the global epidemiology of SSI after appendectomy, despite the increasing number of appendectomies done in the world. The current systematic review and meta-analysis aim at summarising the available data concerning prevalence and incidence of SSI after appendectomy.

\section{REVIEW QUESTIONS}

1. What is the global prevalence of SSI after appendectomy?

2. What is the global incidence of SSI after appendectomy?

\section{OBJECTIVES}

This systematic review and meta-analysis aims to:

1. Determine the global prevalence of SSI after appendectomy.

2. Determine the global incidence of SSI after appendectomy.

\section{METHODS AND DESIGN}

This systematic review and meta-analysis will be reported in conformity with the Preferred Reporting Items for Systematic Reviews and Meta-Analyses (PRISMA) guidelines. ${ }^{18}$ For the present protocol, the Preferred Reporting Items for Systematic Review and Meta-Analysis Protocols (PRISMA-P) for Protocol was used for the reporting. ${ }^{19}$ An additional file shows the PRISMA-P for protocol checklist (see supplementary additional file 1 ).

\section{Criteria for considering studies for the review \\ Types of participants \\ We will include all participants regardless of their country, age and ethnicity.}

\section{Types of studies}

We will include randomised controlled trials, cohort studies, case-control and cross-sectional studies. Only studies reporting the use of Centers for Disease Control and Prevention terminology and, diagnostic criteria for SSI will be considered. ${ }^{20} 21$

Letters to the editor, narrative reviews, commentaries, perspectives and editorials will be excluded.
Types of outcomes

We will consider studies reporting the following outcomes with enough data to compute these estimates:

- Prevalence of SSI after appendectomy.

- Incidence of SSI after appendectomy.

Studies in which relevant data on SSI after appendectomy is impossible to extract even after contacting the corresponding author will be excluded.

\section{Other criteria}

- All published data between 1 January 2000 and 30 December 2017 will be considered.

- No language restriction will be applied.

- For duplicates of studies published in more than one report, the one reporting the largest sample size will be considered.

- Studies with inaccessible full text either online or from the corresponding author will be excluded.

\section{Search strategy for identifying relevant studies}

The search strategy will be conducted in two stages.

\section{Bibliographic database searches}

Relevant articles published on SSI after appendectomy will be identified by searching Excerpta Medica Database (Embase), MEDLINE through PubMed and ISI Web of Science (Science Citation Index) between 1 January 2000 and 30 December 2017, without any language restriction. Text words, Medical Subject Headings terms related to SSI and appendicitis will be used (table 1). When necessary, contact with authors for more information will be made.

\section{Searching for other sources}

We will scan the references of all relevant articles for additional data sources missed during our search, and their full texts will be retrieved. References of pertinent reviews will also be scanned.

\section{Selection of studies for inclusion in the review}

Two reviewers (CD and TNM) will independently evaluate the studies obtained from the searches, using an assessment form to ensure that the selection criteria are reliably applied. These reviewers will screen the titles and

Table 1 Search strategy for PubMed from 1 January 2000 to 30 December 2017

\begin{tabular}{ll}
\hline Search & Search terms \\
\hline$\# 1$ & $\begin{array}{l}\text { Appendectomy OR Appendicectomy OR } \\
\text { Appendices OR "Appendix Epiploica" OR } \\
\text { "Omental Appendix" OR Appendicitis }\end{array}$ \\
\#2 & $\begin{array}{l}\text { "Surgical site infection" OR "Surgical wound } \\
\text { infection" OR "Surgical wound infections" } \\
\text { OR "Surgical site infections" OR "Operative } \\
\text { site infections" OR "Postoperative Wound } \\
\text { Infections" OR "Postoperative Wound Infection" } \\
\text { \#1 AND \#2 Limits: 01/01/2000 to 30/12/2017 on } \\
\text { humans with no language restriction }\end{array}$ \\
\hline
\end{tabular}


abstracts of papers obtained, after which the full texts of potentially eligible papers will be retrieved by at least one reviewer. The two reviewers will independently review the full text of each potentially eligible study, compare their results and resolve any discrepancy by the arbitration of a third reviewer (JNT).

\section{Assessment of methodological quality and reporting of data}

Methodological quality and risk of bias of included studies will be assessed using the tool of bias assessment for prevalence studies developed by Hoy $e t a l^{22}$ and the Cochrane's bias assessment tool for randomised studies. ${ }^{23}$

\section{Data extraction and management}

All references identified after implementation of the searched strategy will be imported inside the Endnote software. All records obtained from various databases will be combined in a single Endnote library, and the duplicates will be noted and removed. A data extraction form will thereafter be used to collect information on the last name of the first author, year of publication, continent, country, study design, study area (rural vs urban), age groups (children or adults), sample size, mean or median age, gender, specific characteristics of the study population, high-risk patients for SSI (patients with diabetes mellitus and HIV/AIDS), clinical type of appendicitis (catarrhal, perforated, suppurated and gangrenous), medical interventions before appendectomy (antibiotherapy and analgesics) healthcare facility (primary or other centre), the surgical method (open surgery or laparoscopy), prevalence and incidence of SSI after appendectomy in the study population. For multinational studies, the prevalence and incidence will be reported for the individual countries. Where it is impossible to disaggregate data of multinational studies by country, the study will be presented as one, and the countries in which the study was done will be reported.

\section{Data synthesis and analysis}

After data collection, a meta-analysis will be conducted. Unadjusted prevalence, incidence and SEs for the study-specific estimates will be recalculated based on the information of crude numerators, and denominators will be provided by individual studies. To keep the effect of studies with extremely small or extremely large prevalence estimates on the overall estimate to a minimum, the variance of the study-specific prevalence/incidence will be stabilised with the Freeman-Tukey double arcsine transformation ${ }^{24}$ before pooling the data using a random effects meta-analysis model. Heterogeneity will be assessed using the $\chi^{2}$ test on Cochrane's $Q$ statistic and quantified by calculating $\mathrm{I}^{2} .{ }^{25}$ Values of $25 \%, 50 \%$ and $75 \%$ for $\mathrm{I}^{2}$ will respectively represent low, medium and high heterogeneity. We will assess the presence of publication bias using funnel plots inspection and Egger's test. ${ }^{26}$ Where substantial heterogeneity will be detected, meta-regression, and subgroup analyses will be performed to investigate the possible sources of heterogeneity using the aforementioned variables and the study quality. In case of substantial clinical heterogeneity, a narrative summary of our findings will be done. The inter-rater agreement for study inclusion between investigators will be assessed using Cohen's $\kappa$ coefficient. ${ }^{27}$ Data analyses will be done using the 'meta' package of the statistical software R (V.3.2.2 (2014-08-14), The R Foundation for statistical computing, Vienna, Austria). This systematic review protocol is registered under the review number: CRD42017070480 in the International Prospective Register of Systematic Reviews. ${ }^{28}$

\section{Presentation and reporting of results}

The study selection process will be summarised using a flow diagram. Quantitative data will be presented in tables of individual studies, in summary tables and forest plots where appropriate. The quality scores and risk of bias for each eligible study will be reported accordingly. This may be tabulated and accompanied by narrative summaries.

\section{Patient and public involvement}

In this study, data will not be collected directly from patients but in published studies available in main databases.

\section{Potential amendments}

Any amendment in the review process will be reported for transparency.

\section{CONCLUSION}

SSI after appendectomy is one of the complications of the surgical treatment of appendicitis. This systematic review aims at providing high-quality evidence on the epidemiology of SSI after appendectomy. We hope this review will help to sensitise surgeons to implement effective strategies to prevent SSI in order to scale down the burden SSI after appendectomy.

\section{Review status}

Preliminary searches.

Author affiliations

${ }^{1}$ Department of Surgery and Specialties, Faculty of Medicine and Biomedical Sciences, University of Yaoundé I, Yaoundé, Cameroon

${ }^{2}$ Department of Internal Medicine and Specialties, Faculty of Medicine and Biomedical Sciences, University of Yaoundé I, Yaoundé, Cameroon

${ }^{3}$ Department of Internal Medicine, Ngong District Hospital, Ngong, Cameroon ${ }^{4}$ Department of Epidemiology and Public Health, Centre Pasteur of Cameroon, Yaoundé, Cameroon

${ }^{5}$ School of Public Health, Faculty of Medicine, University of Paris Sud XI, Le KremlinBicêtre, France

Contributors $\mathrm{CD}$ had the idea. $\mathrm{CD}$ designed and conceived the protocol and drafted the manuscript. TNM, JNT, RNTN and JJB participated in the critical revision of the manuscript for methodology and intellectual content. CD and JJB are the guarantor of the review. All authors approved the final version of this manuscript.

Funding The authors have not declared a specific grant for this research from any funding agency in the public, commercial or not-for-profit sectors.

Competing interests None declared.

Patient consent Not required. 
Provenance and peer review Not commissioned; externally peer reviewed.

Open access This is an open access article distributed in accordance with the Creative Commons Attribution Non Commercial (CC BY-NC 4.0) license, which permits others to distribute, remix, adapt, build upon this work non-commercially, and license their derivative works on different terms, provided the original work is properly cited, appropriate credit is given, any changes made indicated, and the use is non-commercial. See: http://creativecommons.org/licenses/by-nc/4.0/.

\section{REFERENCES}

1. Birnbaum BA, Wilson SR. Appendicitis at the millennium. Radiology 2000;215:337-48.

2. Ansaloni L, Catena F, Coccolini F, et al. Surgery versus conservative antibiotic treatment in acute appendicitis: a systematic review and meta-analysis of randomized controlled trials. Dig Surg 2011;28:210-21.

3. Paajanen $\mathrm{H}$, Grönroos JM, Rautio T, et al. A prospective randomized controlled multicenter trial comparing antibiotic therapy with appendectomy in the treatment of uncomplicated acute appendicitis (APPAC trial). BMC Surg 2013;13:3.

4. Garcell HG, Arias AV, Sandoval CA, et al. Incidence and etiology of surgical site infections in appendectomies: a 3-year prospective study. Oman Med J 2017;32:31-5.

5. Addiss DG, Shaffer N, Fowler BS, et al. The epidemiology of appendicitis and appendectomy in the United States. Am J Epidemiol 1990;132:910-25.

6. de Lissovoy G, Fraeman K, Hutchins V, et al. Surgical site infection: incidence and impact on hospital utilization and treatment costs. Am $J$ Infect Control 2009;37:387-97.

7. Antal A. [Changes in epidemiology, etiology, diagnostics, and therapy of acute appendicitis]. Orv Hetil 2009;150:443-6.

8. Minutolo V, Licciardello A, Di Stefano B, et al. Outcomes and cost analysis of laparoscopic versus open appendectomy for treatment of acute appendicitis: 4-years experience in a district hospital. BMC Surg 2014;14:14.

9. Wei B, Qi CL, Chen TF, et al. Laparoscopic versus open appendectomy for acute appendicitis: a metaanalysis. Surg Endosc 2011;25:1199-208.

10. Long KH, Bannon MP, Zietlow SP, et al. A prospective randomized comparison of laparoscopic appendectomy with open appendectomy: Clinical and economic analyses. Surgery 2001;129:390-400.

11. Margenthaler JA, Longo WE, Virgo KS, et al. Risk factors for adverse outcomes after the surgical treatment of appendicitis in adults. Ann Surg 2003;238:59-66.

12. Berry J, Malt RA. Appendicitis near its centenary. Ann Surg 1984;200:567-75.
13. Leung TT, Dixon E, Gill M, et al. Bowel obstruction following appendectomy: what is the true incidence? Ann Surg 2009;250:51-3

14. Badia JM, Casey AL, Petrosillo N, et al. Impact of surgical site infection on healthcare costs and patient outcomes: a systematic review in six European countries. J Hosp Infect 2017;96:1-15.

15. Perencevich EN, Sands KE, Cosgrove SE, et al. Health and economic impact of surgical site infections diagnosed after hospital discharge. Emerg Infect Dis 2003;9:196-203.

16. Rosenthal VD, Richtmann R, Singh S, et al. Surgical site infections, International Nosocomial Infection Control Consortium (INICC) report, data summary of 30 countries, 2005-2010. Infect Control Hosp Epidemiol 2013;34:597-604.

17. Foster D, Kethman W, Cai LZ, et al. Surgical site infections after appendectomy performed in low and middle human developmentindex countries: a systematic review. Surg Infect 2018;19.

18. Moher D, Liberati A, Tetzlaff J, et al. Preferred reporting items for systematic reviews and meta-analyses: the PRISMA statement. PLoS Med 2009;6:e1000097.

19. Moher D, Shamseer L, Clarke M, et al. Preferred reporting items for systematic review and meta-analysis protocols (PRISMA-P) 2015 statement. Syst Rev 2015;4:1.

20. Horan TC, Andrus M, Dudeck MA. CDC/NHSN surveillance definition of health care-associated infection and criteria for specific types of infections in the acute care setting. Am J Infect Control 2008;36:309-32.

21. Horan TC, Gaynes RP, Martone WJ, et al. CDC definitions of nosocomial surgical site infections, 1992: a modification of CDC definitions of surgical wound infections. Infect Control Hosp Epidemiol 1992;13:606-8.

22. Hoy D, Brooks $P$, Woolf $A$, et al. Assessing risk of bias in prevalence studies: modification of an existing tool and evidence of interrater agreement. J Clin Epidemiol 2012;65:934-9.

23. Higgins JP, Altman DG, Gøtzsche PC, et al. The cochrane collaboration's tool for assessing risk of bias in randomised trials. BMJ 2011;343:d5928.

24. Miller JJ. The inverse of the Freeman - Tukey double arcsine transformation. The American Statistician 1978;32:138.

25. Huedo-Medina TB, Sánchez-Meca J, Marín-Martínez F, et al. Assessing heterogeneity in meta-analysis: $Q$ statistic or 12 index? Psychol Methods 2006;11:193-206.

26. Egger M, Davey Smith G, Schneider M, et al. Bias in meta-analysis detected by a simple, graphical test. BMJ 1997;315:629-34.

27. McHugh ML. Interrater reliability: the kappa statistic. Biochem Med 2012;22:276-82.

28. Danwang C, Temgoua MN, Tochie JN, 2017. Global prevalence and incidence of surgical site infections after appendectomy: a systematic review and meta-analysis protocol. http://www.crd.york ac.uk/PROSPERO/display_record.php?ID=CRD42017075257 